Intraocular currents, Bernoulli's principle and non-drainage scleral buckling for rhegmatogenous retinal detachment

\author{
Abstract \\ For many years, it is not fully understood \\ how non-drainage scleral buckling surgery \\ brings about spontaneous reattachment of the \\ detached retina when retinal breaks remain \\ open at the end of surgery. Various \\ explanations have been put forward, but \\ none more interesting than the effect of fluid \\ currents associated with eye movements. One \\ such explanation involved the physics of the \\ Bernoulli's principle. Daniel Bernoulli was an \\ eighteenth century Swiss mathematician and \\ he described an equation based on the \\ conservation of energy. The sum of pressure \\ energy, potential energy and kinetic energy \\ remains constant. Bernoulli's equation \\ usually applies to closed system such as the \\ flow of fluid through pipes. When fluid flows \\ through a constriction, the speed of fluid \\ increases, the kinetic energy increases. If \\ there was no change in elevation (potential \\ energy), then the increase in kinetic energy \\ must be accompanied by a decrease in \\ pressure energy. In ophthalmic surgery, the \\ Bernoulli's effect is the basis for venturi \\ pumps that drive vitrectomy and \\ phacoemulsification machines. This essay \\ expounds on how Bernoulli's effect might be \\ relevant to scleral buckling for retinal \\ detachment repair. In the era when \\ vitrectomy is increasing the primary surgical \\ operation for retinal detachment, the \\ pervasive advice is to emphasise the \\ importance of patient adopting head posture \\ and remaining still postoperatively. The \\ exception is non-drainage scleral buckling \\ surgery. Early postoperative mobilisation may \\ be vital to achieve reattachment. \\ Eye (2018) 32, 213-221; doi:10.1038/eye.2017.312; \\ published online 19 January 2018
}

D Wong ${ }^{1}$, YK Chan ${ }^{2}$, T Bek ${ }^{3}$, I Wilson ${ }^{4}$ and E Stefánsson ${ }^{5}$

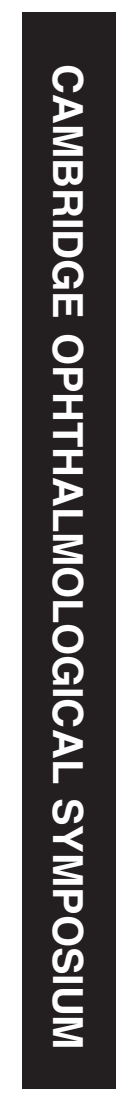

Rhegmatogenous retinal detachment: the role of intraocular currents

In 1984, Robert Machemer attributed the cause of retinal detachment to a combination of dynamic vitreous traction, intraocular current and in the presence of open retinal break or breaks. ${ }^{1}$ Most but not all rhegmatogenous retinal detachments are associated with posterior vitreous detachment (PVD). ${ }^{2}$ PVD is usually a precondition for the development of U-tears. ${ }^{3}$ Tears are formed at the posterior border of the vitreous base because of dynamic traction. Dynamic traction is caused by eye (and some believe head) movements. ${ }^{4}$ Saccadic and pursuit eye movements are usually rotational and can reach high angular velocities. ${ }^{5}$ In the presence of a PVD, the content of the vitreous cavity no longer move bodily as a single semi-solid structure. ${ }^{4}$ Being attached at the vitreous base, the anterior vitreous gel continues to move at roughly the same angular velocity as the eye wall. In contrast, being detached posteriorly, the posterior vitreous gel lags somewhat behind (Figure 1). The weight of the vitreous and angular momentum generated by rotational eye movements give rise to dynamic traction. As dynamic traction is exerted at the vitreous base, this is generally the location for the development of U-tears. Because the direction of traction is from posterior to anterior, the two horns of the U-tear are directed anteriorly.

The presence of retinal breaks however, may or may not be sufficient for the development of retinal detachment. With PVD, the retrohyaloid space is occupied by aqueous. Because this is a liquid with low adherence to the eyewall, little of the angular momentum of rotational eye movement is transferred to the retrohyaloid fluid. The fluid tends to stay stationary and to lag behind eye movements but continue in the Liverpool, UK

\footnotetext{
2Department of Mechanical Engineering, University of Hong Kong, Hong Kong SAR, China

${ }^{3}$ Department of Clinical Medicine, Aarhus University, Aarhus, Denmark

${ }^{4}$ Department of Chemical Engineering and Biotechnology, University of Cambridge, Cambridge, UK

${ }^{5}$ Landspitali University Hospital, University of Iceland, Reykjavík, Iceland

Correspondence: D Wong, St Paul's Eye Unit, Royal Liverpool University Hospital, Prescot St, Liverpool L7 8XP, UK Tel: +44 7940018 6422; Fax: +44 1517065905. E-mail: shdwong@gmail. com

Received: 14 November 2017

Accepted: 30 November 2017

Published online:

19 January 2018
}

${ }^{1}$ Royal Liverpool University Hospital, 

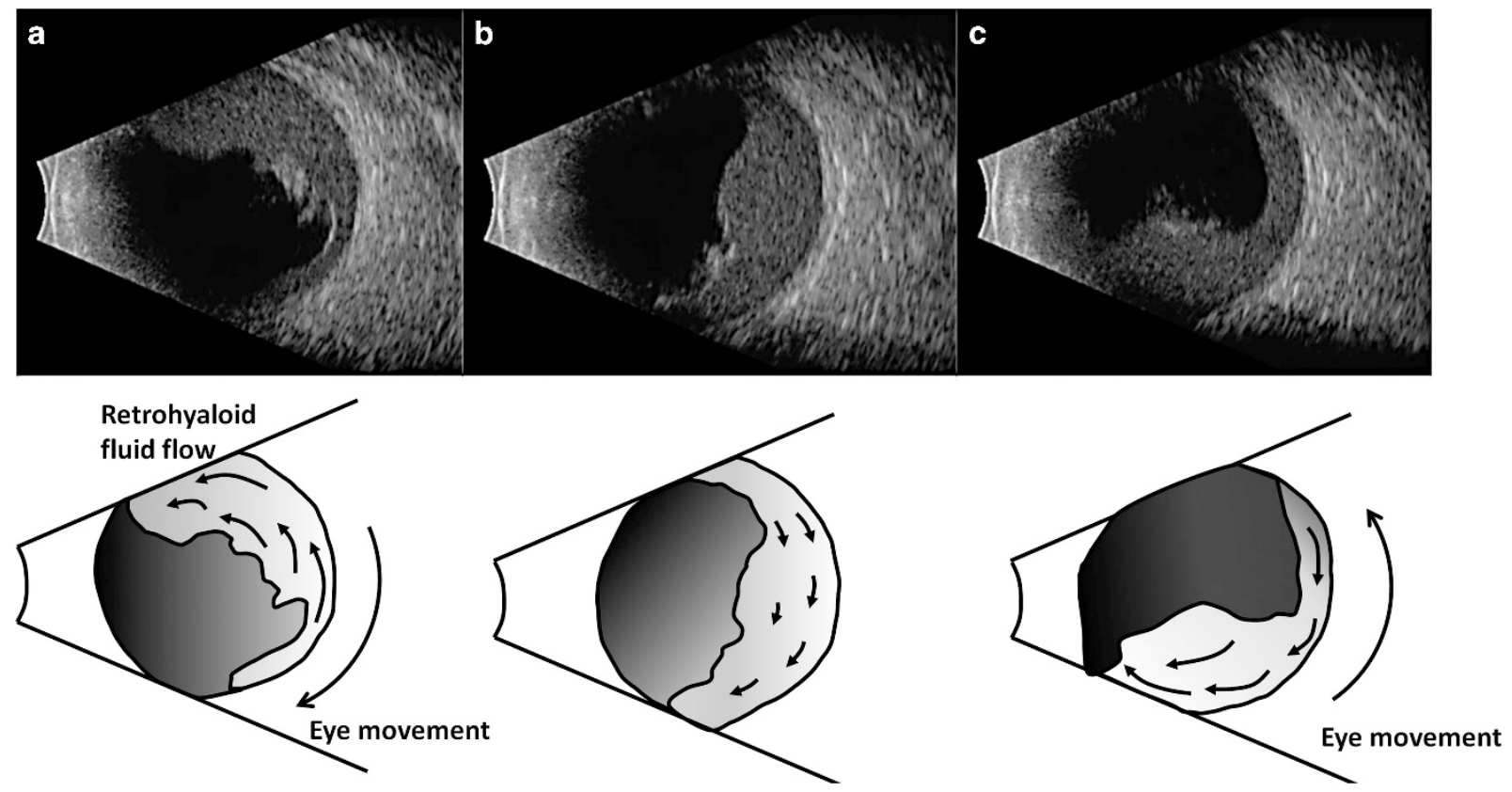

Figure 1 Horizontal B-mode ultrasound of patient with retrohyaloid blood. Patient looking right and left. The vitreous gel is dark highlighted by the retrohyaloid blood. The vitreous gel taking on a sinusoidal whip-like motion, (a) firstly being concave upwards, (b) adopting an intermediate position, and (c) then concave downwards. This is dynamic traction, which is the pull of the vitreous on the retina at the posterior border of the vitreous base that may cause retinal tear. The retrohyaloid fluid flow is shown by the movement of the fine echo of the retrohyaloid blood. Both the motion of the vitreous gel and the flow of fluid are clearly visible on the video, as shown in Supplementary file. The retrohyaloid fluid is impinging on angle between the vitreous and the retina, as shown in a. In the presence of a U-tear, such fluid current is channelled by the vitreous into the subretinal space via an open retinal break.

same direction when the rotation stops (Figure 1b). The retrohyaloid fluid seemingly 'flow' firstly, in the opposite direction to rotational eye movement when the eye moves and subsequently, in the same direction when the eye stops (Supplementary Video 1). This creates a sinusoidal whip-like movement of the detached vitreous gel which can be clearly seen on ultrasonography (Figure 1c). The 'flow' of course is relative. Whether it is the eye wall that moves whilst the fluid remain stationary or whether it is retrohyaloid fluid that flows whilst the vitreous remains stationary, the net result is the same. The retrohyaloid fluid would impinge at the posterior border of the vitreous base where the vitreous gel remains attached. In the presence of a retinal break, the fluid would be channelled under the retina causing a detachment of the retina. Probably because of intraocular currents, all retinal detachment firstly lifts off anterior to the U-tear and the subretinal fluid subsequently spread posteriorly towards the macula and the optic disc. This is illustrated in

Figure 2 that shows snapshots of the B-mode ultrasound of a retinal detachment with a wave of subretinal fluid propagating posteriorly.

This is the traditional explanation for the development of rhegmatogenous retinal detachment. The caveat is that some retinal detachment occurs without posterior vitreous detachment. ${ }^{6}$ A retinal detachment arising from dialysis seldom presents with PVD. ${ }^{7}$ By definition, the vitreous is attached anterior and posterior to the dialysis. ${ }^{8}$ Pigment clumps are often observed in anterior vitreous indicating that there may be a separation anteriorly of the vitreous base as well as a dialysis in the retina. ${ }^{9}$ Retinal detachments arising from round atrophic holes or outer leaf breaks in schisis are usually not accompanied by PVD. ${ }^{10}$ In some of these cases, the vitreous gel may be synergistic with pockets of liquefied vitreous which may be sufficient to cause the vitreous gel not to move bodily as a semi-solid gel. ${ }^{11}$ In which case, the relative movement may be sufficient to cause traction or induce currents that cause retinal detachment.

The aim of any retinal detachment treatment is to close retinal breaks. There are two manoeuvres that would achieve this objective. They are namely, scleral buckling (sometimes referred to as external tamponade) or internal tamponade. Let us examine both situations.

\section{Scleral buckle}

A scleral buckle pushes the eye wall inwards and consequently approximates the retinal pigment epithelium towards the retinal break. The first scleral buckling performed with a retained explant was introduced by Ernst Custodis in 1949. ${ }^{12}$ Prior to this 


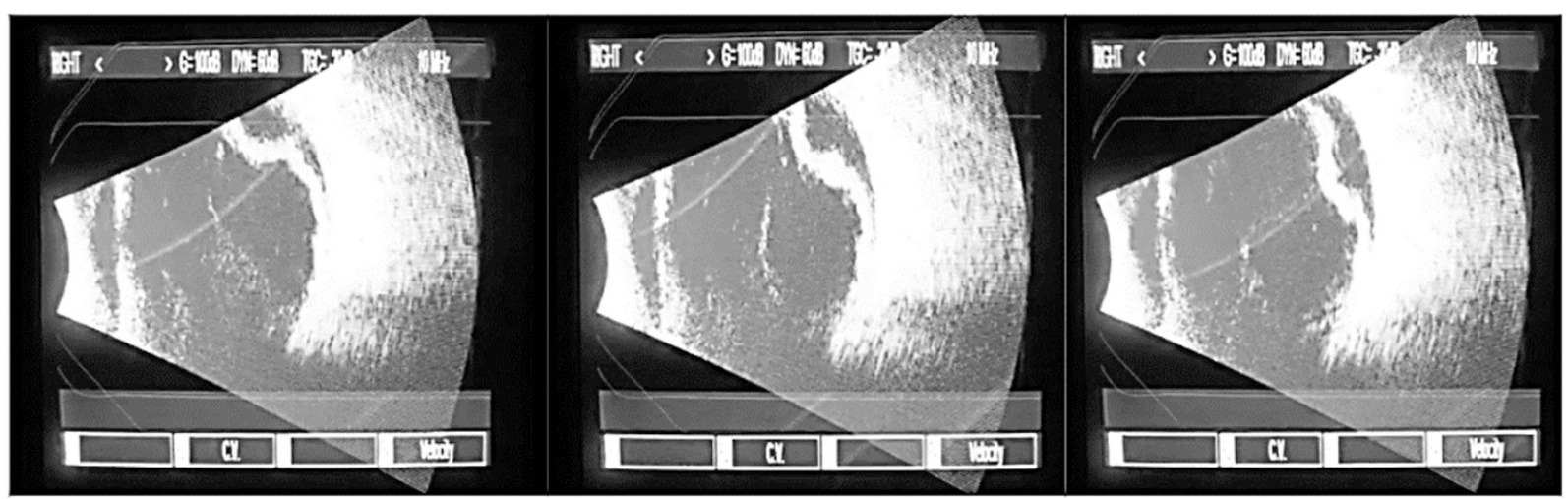

Figure 2 Horizontal B-mode ultrasound of a patient with a retinal detachment. Patient looking left and right. Note the subretinal fluid initially situated peripherally propagated posteriorly as a wave. The vitreous again has the same sinusoidal motion instigated by the eye movement.

different forms of scleral buckling was achieved by scleral resection, ${ }^{13}$ imbrication ${ }^{14}$ or suturing. ${ }^{15}$ The aim of such surgery is ostensibly to close the retinal break by opposing whole thickness of the eyewall, including the sclera, choroid and retinal pigment epithelium, to the retinal break. In other words, the indent is such that the retinal break is now occluded. One of the three conditions listed by Machemer ${ }^{1}$ as being necessary for retinal detachment that is, the open break, would no longer be present. Retinal reattachment occurs despite the fact that the dynamic traction and intraocular currents are still persist. In 1965, Harvey Lincoff demonstrated that scleral buckling without drainage of subretinal fluid was compatible with success. ${ }^{16}$ Selecting the appropriate patients for non-drainage surgery becomes a much discussed topic. ${ }^{17}$ Drainage creates hypotony such that a high indent can be achieved. Without drainage surgery, scleral buckle merely approximates the break to the indent but often does not close the retinal break. The retina remains detached at the end of surgery indeed can remain detached for some days postoperatively. The question of how reattachment can occur in the presence of opened retinal breaks is the subject of this essay.

One additional manipulation that surgeons carried during scleral buckling is cryotherapy (and in the past, full thickness diathermy). ${ }^{18}$ Cryotherapy has been shown as a risk factor for development of proliferative vitreoretinopathy (PVR). ${ }^{19}$ Therefore historically, there was a period when scleral buckling without drainage of subretinal fluid and without cryotherapy were in vogue to reduce PVR. The success rate of such so-called 'minimal surgeries' were shown in several series to be just as high as that with cryotherapy. ${ }^{20}$ We can therefore eliminate as a possibility that retinopexy (whether by cryotherapy or full thickness diathermy) is in some way causing reattachment of the retina. Indeed, one of us (DW) carried out 10-year follow-up of a cohort of patients treated with buckling and no cryotherapy. The retina of most patients remain attached. Retinal re-detachment was a late and infrequent complication that occurred only in patients with reduction of buckle height months or years following the initial surgery. ${ }^{21}$ As reduction in buckle height with time is probably inevitable in a small proportion of patients, it was recommended that cryotherapy be performed with the initial surgery. Although this is the standard approach, there are those who still recommend postoperative laser after the retina has reattached before the application of retinopexy. This may or may not reduce the risk of PVR, but not performing retinopexy at the time of surgery in our opinion unequivocally rule out retinopexy as a cause of reattachment.

\section{How does scleral buckle with unclosed hole achieve retinal reattachment?}

For some time, the cause of the r eattachment in nondrainage scleral buckling with open retinal breaks remains unclear. It has been a subject of much speculation. Some argued that dynamic traction is reduced. In the case of radial indentation, buckling over the break brings the vitreous base closer to the centre of the eye. Dynamic traction at the break would be transferred to the vitreous base of the retina immediately adjacent to the break. ${ }^{9}$ Reduction of dynamic traction alone may help retinal reattachment. If there was no intraocular current channelling pre-retinal fluid through the retinal break, the pump action of the retinal pigment epithelium (RPE) may be sufficient to tip the balance in favour of retinal reattachment. There is however, no reason to believe that intraocular currents would stop, unless both eyes are at rest. Indeed bed rest had been recommended by Stellwag in 1881 and Donders in 1886 as 
a treatment for retinal detachment before more effective methods were introduced. ${ }^{22}$

\section{A physical model}

In 1986, Clemens et al ${ }^{23}$ using a physical model of retinal detachment showed how intraocular currents alone could bring about retinal reattachment with opened retinal breaks. The evidence was convincing because it was a physical model which could be visualised. The model used a fish tank within which was a membrane that separated the fluid into a 'preretinal' and a 'subretinal' compartment. There was a hole in the membrane. The tank rested on rollers which enabled the whole tank to rock backwards and forwards. The motion was designed to induce a current flowing over the retinal hole. The authors showed that if a cylinder was placed directly underneath the hole, the current eventually cause the fluid from the 'subretinal' compartment to flux through the hole into the 'preretinal' space such that eventually the membrane draped over the bottom of the tank and the cylinder-mimicking retinal reattachment (Figure 3). They coloured the fluid in the 'subretinal' compartment with ink such that with each backwards and forwards movement of the tank, puffs of colour fluid were seen to egress from the hole. What makes the whole model all the more convincing was the fact that the cylinder had to be placed directly under the hole. If it was placed anywhere else, the reattachment of the membrane did not occur. The authors did not elucidate the mechanism of the reattachment in the model. They attempted to perform qualitative and quantitative studies of their model using finite element analysis. The impact of the model from Clemens et al was to change clinical practice. Having visualised the spontaneous reattachment in the model, most surgeons are persuaded that movement rather than

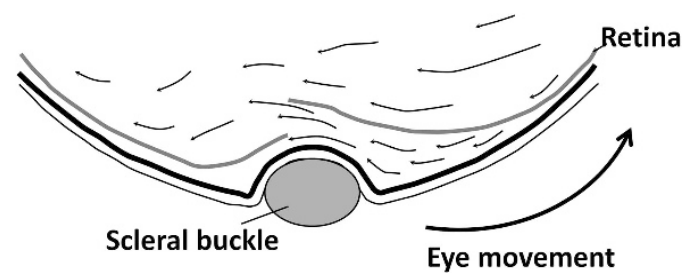

Figure 3 Possible effusion of subretinal fluid into the vitreous space during eye movements. The motion of the eye to the right will generate pressure on the membrane from the super-retinal fluid as it tries to make the super-retinal fluid move (equal and opposite forces). This will generate pressure on the sub-retinal fluid and force it out of the break (as well as the lower pressure in the super-retinal fluid). A high density of arrows indicates a high flow rate. The higher flow rate leads to a drop of pressure near the scleral buckle. The detached retina near the tear may be forced downwards, extracting the subretinal fluid. bed rest helps resolution of subretinal fluid. This is the usual advice given to patients who received non-drainage surgery resulting in opened retinal breaks. There was however, no mention of the Bernoulli effect by Clemens et al.

An example of a patient that had non-drainage scleral buckling surgery is shown in Figure 4. The depth of subretinal fluid was such that the retinal break was opened at the end of surgery (Figure 4a). Postoperatively, the patient took it upon herself to 'posture', staying very upright and still. The retina remained detached for 10 days when she attended clinic. The patient was advised to resume normal physical activity and to take a daily stroll. A week later, the retina was totally reattached without further treatment, as shown on the right.

\section{Bernoulli's principle}

Bernoulli's principle is based on the axiom of preservation of energy. In flowing fluid, the total energy is the sum of kinetic and static energy. Kinetic energy is a function of the velocity and is expressed $m v^{2}$, where $m$ is mass and $v$ is velocity. Static energy is a function of height and pressure. Assuming height is constant, static energy is a function of the pressure of the fluid. In simple terms this means that if the velocity of a liquid or gas was increased the pressure would go down. This is indeed the principle used for flying, where air passing over a wing has a longer distance to and has a higher speed, resulting in lower air pressure over the wing compared with below the wing, thereby generating lift. This is also the principle used to create suction in laboratories and operating rooms. It is sometimes referred to as the Venturi pump where rapidly flowing water or air through a constriction produces a local low pressure that can be harnessed for suction of fluids.

\section{Finite element analysis}

Nearly a quarter of a century later, William Foster and his colleagues revisited Clemens model. They used finite element analyses and considered the current flowing over an indent within the tank. ${ }^{24}$ They considered the nature of the membrane and considered the case when it was either flexible or stiff. At the same time, they estimated the velocity of the current over the indent and the hole in the membrane and the pressure in different parts of the model. These authors were the first to suggest that the fast current over the indent could be associated with lower pressure within the system, thus acting as a crude pump. Their model demonstrated that with fluid flow, the pressure was increased in front of the buckle and lower behind the buckle. Within the 'subretinal space' the increased pressure on one side of the indent led to fluid 

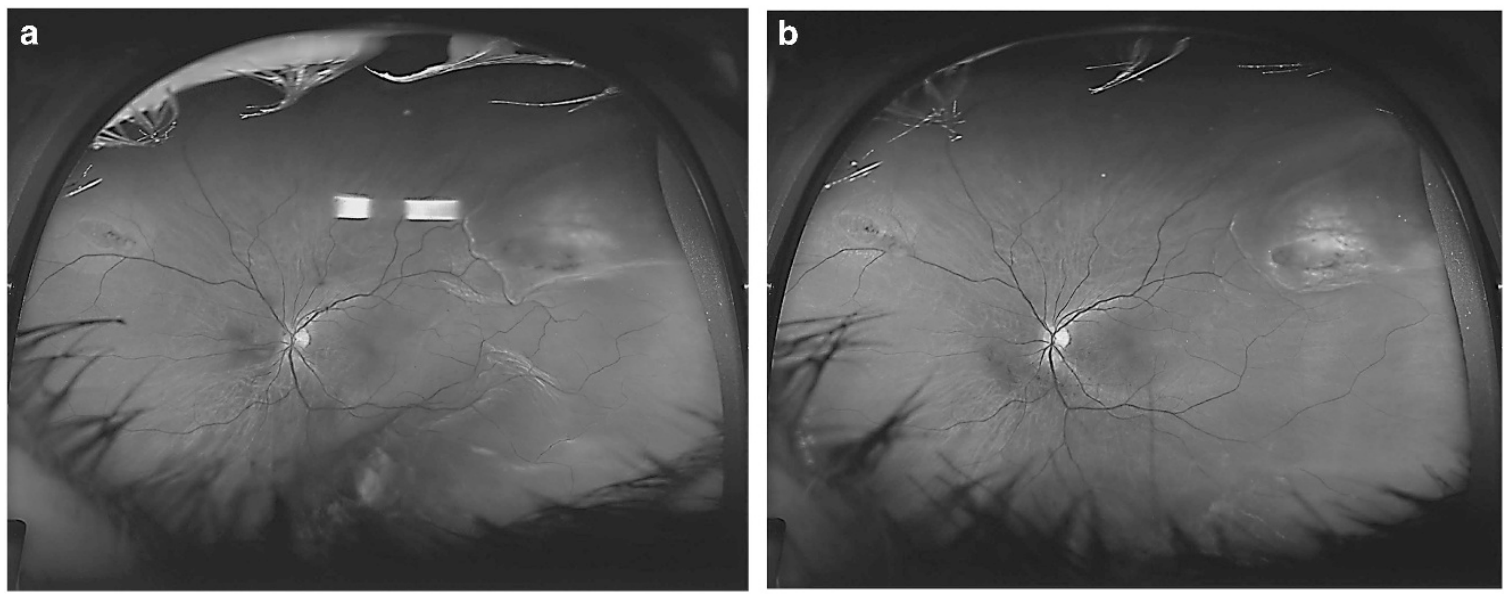

Figure 4 Fundus photograph of a patient treated with non-drainage scleral buckling surgery 10 days postoperatively, as shown in (a). The patient took it upon herself to 'posture' and kept quite still. The patient was advised to resume normal activity and exercise gently by taking a daily stroll. A week later, the retina was totally reattached without further treatment, as shown in (b).

egressing out through the hole and on the decreased pressure on the lee side of the indent led to re-apposition of the membrane to the bottom of the tank. They compared different types of eye movements and the flow currents that they generated. Their simulation showed that rapid eye movement (REM) was most efficient in removing fluid from the subretinal space. It was envisaged that fluid flow with eye movements over an indent can cause retinal reattachment (Figure 5).

Recent work in the University of Liverpool compared the effect of head and eye movements in a computer model. The results showed that certain head movements may also be as important as rotational eye movement in generating traction forces to cause extension of retinal detachment (personal communication). ${ }^{25}$

\section{Intraocular currents in vitrectomised eyes with gas or silicone oil fill}

With vitrectomy, theoretically dynamic traction is removed. In most surgeries, usually complete drainage of subretinal fluid is attempted. The vitreous or preretinal fluid is replaced by an air bubble. The buoyancy of an air bubble meant that most of the upper retina would be reapposed to the underlying RPE. The surface tension of air prevents fluid regaining access to the subretinal space through retinal breaks. In other words, retinal reattachment is achieved via internal tamponade. To increase the longevity of the internal tamponade effect, different inert gas mixtures or sometimes silicone are used to replace air in the vitreous cavity.

The presence of internal tamponade agents be it gas or oil ideally should totally fill the vitreous cavity. In practice, total fill is difficult to achieve. ${ }^{26}$ This difficulty is due to the fact that the retinal surface is hydrophilic. As such, gas (all types, including air) and silicone oil form an obtuse contact angle with the retina. ${ }^{27}$ Practically, part of the volume of the tamponade goes to form the meniscus which has no tamponade effect. The result is that a slight under-fill would leave a large area of the retina not in contact with the tamponade agent. Silicone oil has a specific gravity of (0.97) close to that of water (1.00) and is hydrophobic. Consequently, the contact angle and the meniscus are both large. When silicone oil is used therefore, there is a film of aqueous fluid around most of the silicone oil tamponade. In the case of gas, absorption of the gas results in a progressively smaller intraocular bubble and an increasing large pool of aqueous beneath the bubble.

In the past, some of us have studied the movement of the tamponade inside the eye with simulated saccadic eye movements. We used a stepper motor driven by a computer programme to mechanically rotate a model eye chamber containing aqueous and air or silicone (Figure 6a). ${ }^{28}$ The model eye chamber was surface modified to mimic the vitreoretinal interface. ${ }^{26}$ The movement of the bubble of tamponade agents were recorded by video. The videos showed that there was always a lag in which the movement of the chamber was followed a little later by movement of the tamponade. This lag depended on the viscosity of tamponade agent. For a low viscosity fluid like air, the lag was minimal. It was possible to analyse the video and to measure the relative angular movement and velocity of the bubbles (and by inference the fluid beneath the bubble). In other words, the fluid flowed almost but not quite at the same speed as the eyewall (Figure 6b). ${ }^{29,30}$ In eyes with tamponades, there was aqueous under bubble and there 


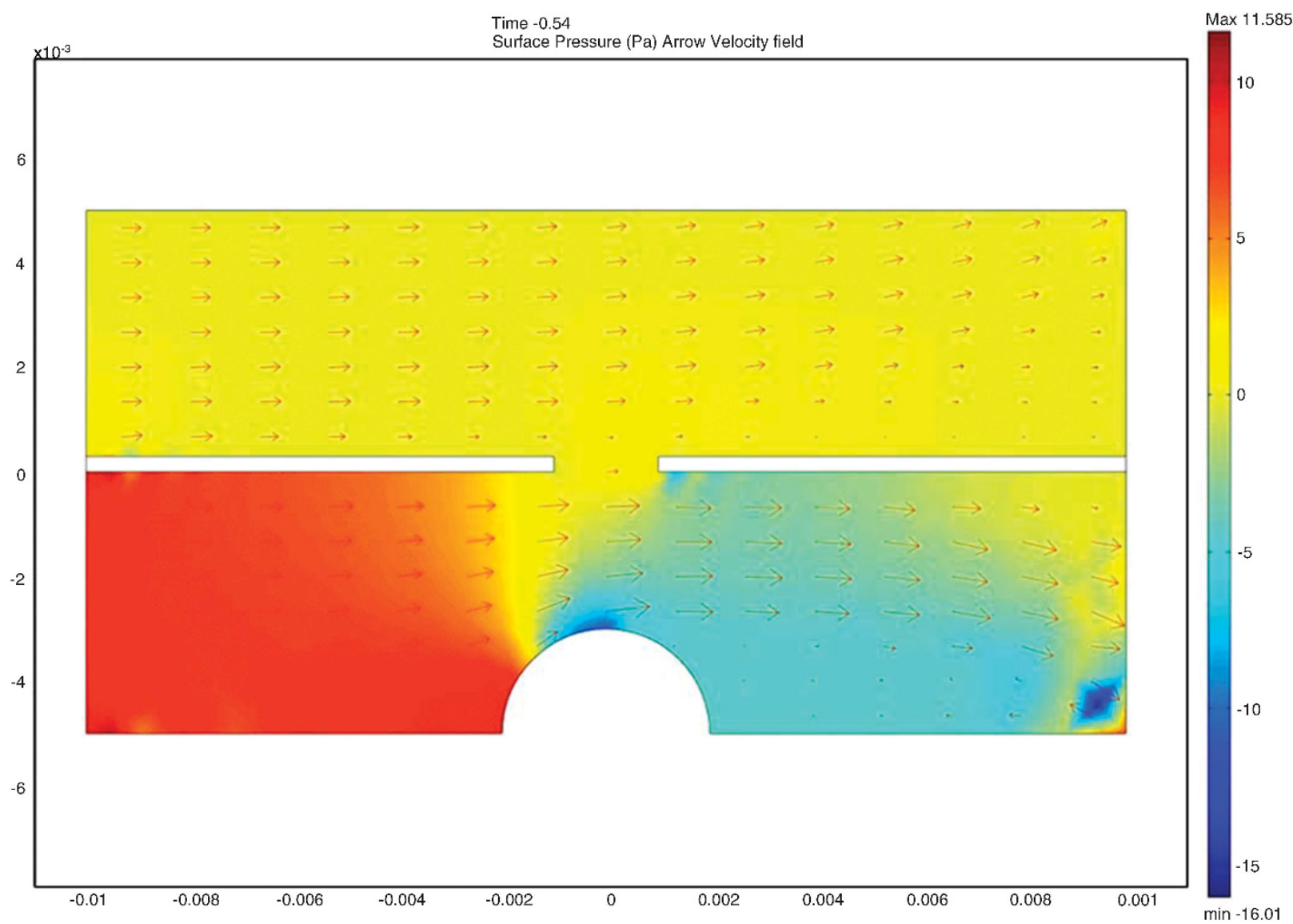

Figure 5 Simulated fluid flow from left to right. ${ }^{24}$ The length of the arrows indicated the magnitude of the speed of the flow. The colour indicates the pressure level, with the highest indicating with red colour, and the lowest with blue colour. Reproduced with permission of Graefe's Archives of Clinical and Experimental Ophthalmology.

was movement or flow in the aqueous. This flow was relative (it was actually the eyewall that was moving rather than the aqueous flowing). Vitrectomy may relieve dynamic traction. The model clearly showed that the use of tamponade, unless the fill is total, does not eliminate intraocular currents (Figure 6c). ${ }^{29,30}$

\section{How does inferior retinal breaks stay closed without scleral buckle}

The tamponade effect to inferior breaks becomes a contentious subject. The orthodox approach was to combine vitrectomy with scleral buckling. It was thought that a shrinking gas bubble could not be relied upon to tamponade inferior breaks for any length of time. Equally, it was awkward for patients to posture their heads and to use the bubble to support inferiorly situated retinal breaks or so it was thought. In 2004, this concept was challenged by the simultaneous publications in the same edition of British Journal of Ophthalmology by teams at St Thomas' Hospital and Moorfields Eye Hospital. ${ }^{31,32}$ Both groups found that vitrectomy and gas alone for retinal detachment with inferior retinal breaks had an acceptable success rate. Both concluded that scleral buckling and its complications could be avoided. In the St Thomas' case series, patients were asked to posture face up or alternative left or right side down for one week. In the Moorfields' series, the patients were also instructed to posture alternating, left or right cheek down depending on the location of the breaks. Both were real life and pragmatic case series. Both showed that without the use of buckle retinal reattachment could also be achieved. They did not speculate, beyond alluding to the fact that gas tamponade could disrupt intraocular currents, as to how inferior retinal breaks could be closed.

There are a number of possibilities. Firstly, though awkward and difficult, head posture by the patients might be effective and achieve the desired tamponade effect. Secondly, the retinal breaks might be closed peroperatively or shortly afterwards. Internal tamponade is only required until retinopexy achieve a watertight seal. Using laser, Martinez has shown that in selected cases 


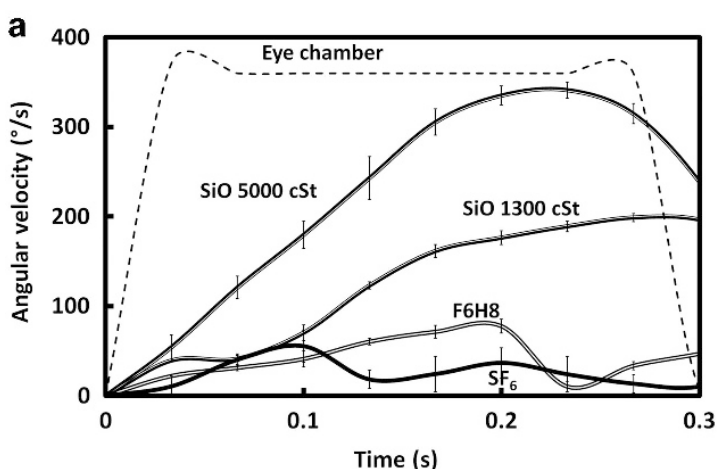

C

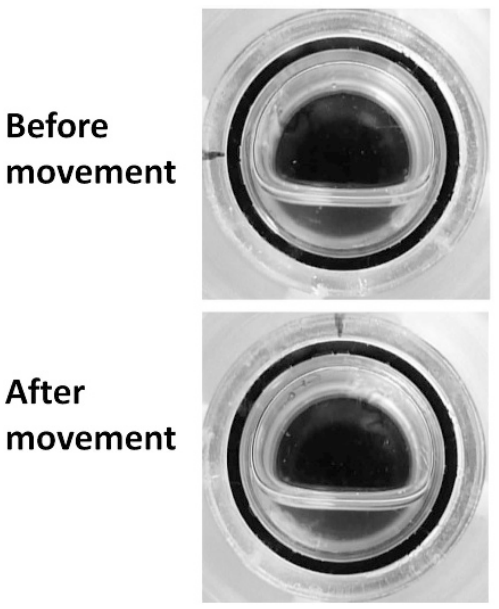

F6H8

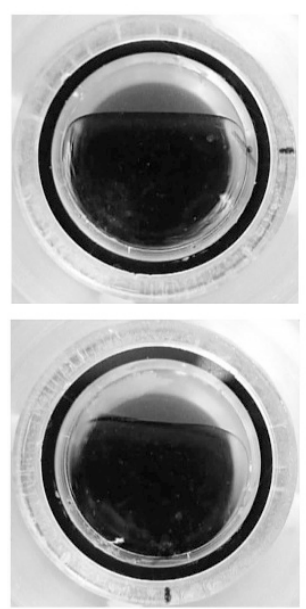

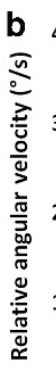

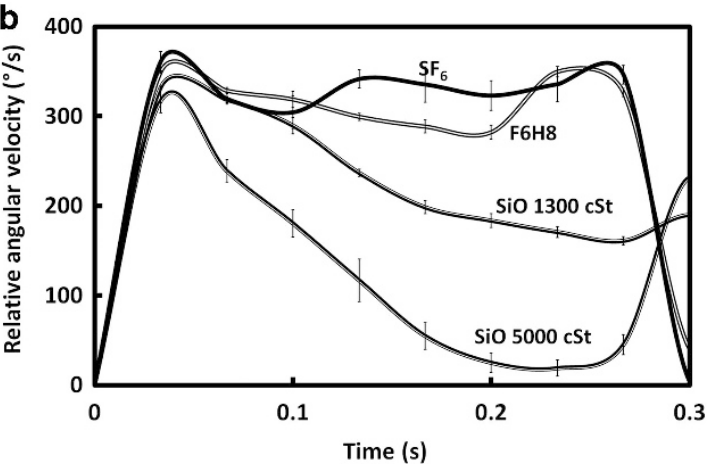

SiO 1300 cSt

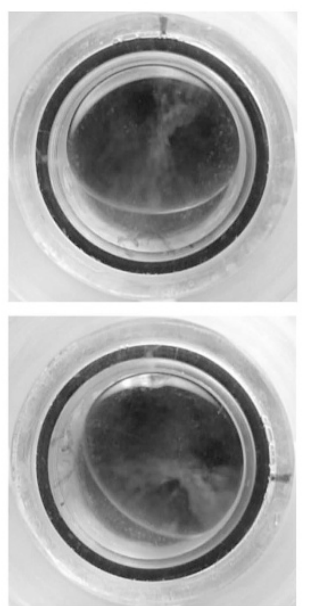

SiO $5000 \mathrm{cSt}$

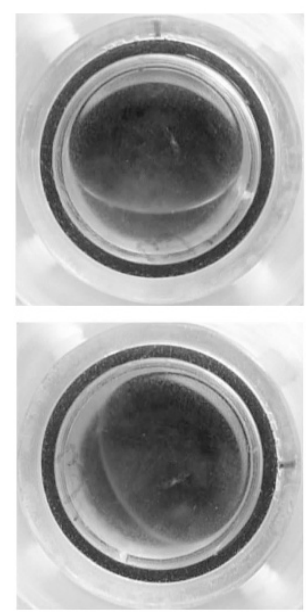

Figure 6 (a) The position of the various tamponade agents before and immediately after the rotation of the chamber. Noted that the chamber has been rotated for $90^{\circ}$, as indicated by the line mark. (b) Angular velocity of various tamponade agents during the rotation of the eye chamber. The dash line corresponds to the motion of the eye chamber. (c) The angular velocity of the various tamponade agents relative to the eye chamber. The higher the viscosity of the tamponade agent, the lower the relative angular velocity. The higher the viscosity of the tamponade agents, the more the angular displacement of the tamponade, thus a smaller relative motion between the tamponade agent and the chamber. ${ }^{28,29}$

with inferior retinal breaks, gas tamponade was not necessary. ${ }^{33}$ For the vast majority of cases without epiretinal membrane, perhaps a few day of tamponade may be all that is needed for achieve adhesion. Thirdly, the presence of a large size bubble may reduce the aqueous compartment. The aqueous compartment although present may be too small to generate any significant current or bulk flow. Machemer made similar observations in diabetic proliferative retinopathy with tractional retinal detachment (TRD). ${ }^{1}$ Even though there could be full thickness retinal breaks within areas of TRD, they did not cause rhegmatogenous retinal detachments until the membranes were excised by vitrectomy. He speculated that the intraocular currents within these small compartments formed by the membranes were not sufficient to cause extension of localised retinal detachment. Fourthly, the combination of vitrectomy and tamponade means that dynamic traction is no longer prominent. All these combined may be sufficient to achieve a relatively high success rate.

Some surgeons however still hold the view that scleral buckling continues to be important. There are clinical evidences especially from more complex cases involving proliferative vitreoretinopathy that scleral buckling for inferior breaks were associated with higher success rate than vitrectomy and tamponade alone. ${ }^{34-36}$ It could be argued that a gaseous tamponade bubble eventually would get smaller. Any retinal breaks not sealed by retinopexy might cause retinal re-detachment of the retina. Indeed, in recurrent retinal detachment Lincoff suggested that responsible retinal break would be found at the lower edge of the shrinking bubble. ${ }^{37}$ Such a case is presented in Figure 7 with an unsealed break at 9:30 o/c causing recurrent retinal detachment when the gas bubble became smaller. 


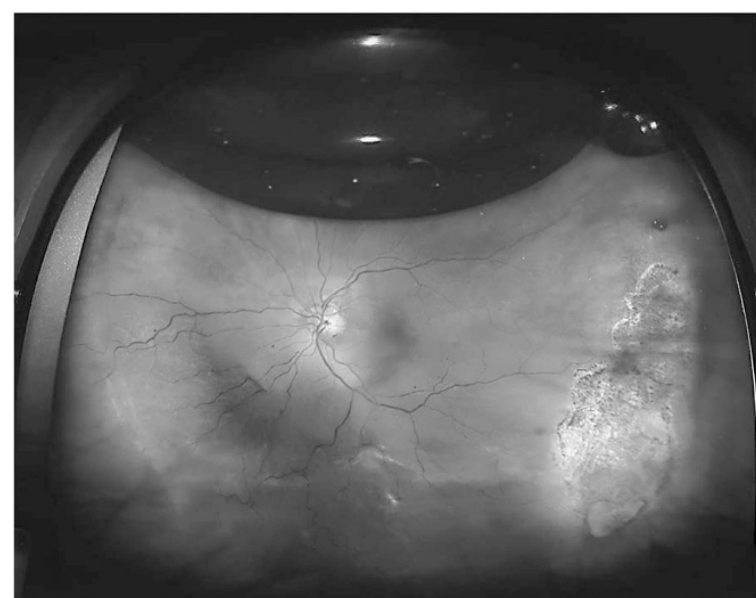

Figure 7 Fundus photograph of a patient treated with primary vitrectomy without scleral buckling. The image shows the recurrence of the retinal detachment when the bubble is absorbed. The fluid is higher nasally and suspected unsealed break is found at 9:30 o/c. Lincoff ${ }^{36}$ suggested the level of meniscus when redetachment first occurred would indicate the location of the unsealed break.

\section{Early mobilisation for some patients}

From what we understand, intraocular currents are important in the formation of retinal tears and in the initiation of retinal detachment. In the presence of an appropriate placed scleral buckling, intraocular fluid flowing over an indent can help reattach the retina. Bernoulli's principle helps us to understand how the motion of the liquid causes this to happen: in practice the dynamics of the fluid are more complex that the simple Bernoulli approach and the equations of motion (the Navier-Stokes equations) have to be solved for the fluids and the interfaces. In vitrectomised eyes, although controversy exists as to the need for scleral buckling, there is no doubt that intraocular currents are also present when the bubble of tamponade gets smaller. The trend is for more vitrectomies and fewer scleral buckling procedures to be performed for repairing retinal detachment. The emphasis of ward staff, nurses and resident doctors has been focused on advising patient to posture and to keep still. This advice is further reinforced by information published in leaflets and posture devices advertised on the internet. For the minority of patients treated with scleral buckling especially without drainage of subretinal fluid, early mobilisation after surgery may be helpful rather than harmful. This message could be in danger of being lost from our collective consciousness because of the pervasive advice on posturing with vitrectomy.

\section{Conflict of interest}

The authors declare no conflict of interest.

\section{References}

1 Machemer R. The importance of fluid absorption, traction, intraocular currents, and chorioretinal scars in the therapy of rhematogenous retinal detachments. XLI Edward Jackson Memorial Lecture. Am J Ophthalmol 1984; 98: 681-693.

2 Johnson MW. Posterior vitreous detachment: evolution and complications of its early stages. Am J Ophthalmol 2010; 149 (3): 371-382.e371.

3 Sharma S, Walker R, Brown GC, Cruess AF. The importance of qualitative vitreous examination in patients with acute posterior vitreous detachment. Arch Ophthalmol 1999; 117(3): 343-346.

4 Repetto R, Tatone A, Testa A, Colangeli E. Traction on the retina induced by saccadic eye movements in the presence of posterior vitreous detachment. Biomech Model Mechanobiol 2011; 10(2): 191-202.

5 Knox PC, Weir CR, Murphy PJ. Modification of visually guided saccades by a nonvisual afferent feedback signal. Invest Ophthalmol Vis Sci 2000; 41(9): 2561-2565.

6 Akiba J, Konno S, Yoshida A. Retinal detachment associated with a macular hole in severely myopic eyes. Am J Ophthalmol 1999; 128(5): 654-655.

7 Kennedy CJ, Parker CE, McAllister IL. Retinal detachment caused by retinal dialysis. Aust N Z J Ophthalmol 1997; 25(1): 25-30.

8 Hagler WS. Retinal dialysis: a statistical and genetic study to determine pathogenic factors. Trans Am Ophthalmol Soc 1980; 78: 686-733.

9 Chignell AH, Wong D. Management of Vitreo-Retinal Disease: A Surigcal Approach. Springer, 1999.

10 Ung T, Comer MB, Ang AJ, Sheard R, Lee C, Poulson AV et al. Clinical features and surgical management of retinal detachment secondary to round retinal holes. Eye (Lond) 2005; 19(6): 665-669.

11 Kuhn F, Aylward B. Rhegmatogenous retinal detachment: a reappraisal of its pathophysiology and treatment. Ophthalmic Res 2014; 51(1): 15-31.

12 Custodis E. [Treatment of retinal detachment by circumscribed diathermal coagulation and by scleral depression in the area of tear caused by imbedding of a plastic implant]. Klin Monbl Augenheilkd Augenarztl Fortbild 1956; 129(4): 476-495.

13 Meek RE. Meridional lamellar scleral resection, in separation of the retina. Am J Ophthalmol 1954; 38(6): 777-780.

14 Swan KC. Scleral imbrication for retinal detachment. AMA Arch Ophthalmol 1959; 61(1): 110-114.

15 Castroviejo R. A new approach for sclera-shortening procedure. AMA Arch Ophthalmol 1956; 55(4): 572-575.

16 Lincoff HA, Baras I, Mc LJ. Modifications to the custodis procedure for retinal detachment. Arch Ophthalmol 1965; 73 (2): 160-163.

17 Leaver PK, Chignell AH, Fison LG, Pyne JR, Saunders SH. Role of non-drainage of subretinal fluid in re-operation for retinal detachment. Br J Ophthalmol 1975; 59(5): 252-254.

18 Kang SW, Kim JH, Shin WJ, Kim JI. Subretinal fluid bleb after successful scleral buckling and cryotherapy for retinal detachment. Am J Ophthalmol 2008; 146(2): 205-210.e201.

19 Nagasaki H, Shinagawa K, Mochizuki M. Risk factors for proliferative vitreoretinopathy. Progress Retin Eye Res 1998; 17(1): 77-98.

20 Chignell AH, Markham RH. Retinal detachment surgery without cryotherapy. Br J Ophthalmol 1981; 65(5): 371-373. 
21 Deutsch J, Aggarwal RK, Eagling EM. Removal of scleral explant elements: A 10-year retrospective study. Eye 1992; 6 (6): 570-573

22 Rezaei KA, Abrams GWThe history of retinal detachment surgeryIn:Kreissig I(ed) Primary Retinal Detachment. Springer: Berlin, Heidelberg, 2005.

23 Clemens S, Kroll P, Stein E, Wagner W, Wriggers P. Experimental studies on the disappearance of subretinal fluid after episcleral buckling procedures without drainage. Graefes Arch Clin Exp Ophthalmol 1987; 225(1): 16-18.

24 Foster W, Dowla N, Joshi S, Nikolaou M. The fluid mechanics of scleral buckling surgery for the repair of retinal detachment. Graefes Arch Clin Exp Ophthalmol 2010; 248(1): 31-36.

25 Personal communications from Vroon, J. Deft University of Technology, December 2017.

26 Fawcett IM, Williams RL, Wong D. Contact angles of substances used for internal tamponade in retinal detachment surgery. Graefes Arch Clin Exp Ophthalmol 1994; 232(7): 438-444.

27 Hillier RJ, Stappler T, Williams RL, Turner GS, Wong D. The impact of axial length on retinal tamponade for gas, silicone oil, and heavy silicone oil, using an in vitro model. Graefes Arch Clin Exp Ophthalmol 2011; 249(5): 671-675.

28 Chan YK, Ng CO, Knox PC, Garvey MJ, Williams RL, Wong D. Emulsification of silicone oil and eye movements. Invest Ophthalmol Vis Sci 2011; 52(13): 9721-9727.

29 Chan YK, Williams RL, Wong D. Flow behavior of heavy silicone oil during eye movements. Invest Ophthalmol Vis Sci 2014; 55(12): 8453-8457.
30 Chan YK, Cheung N, Wong D. Factors influencing the shear rate acting on silicone oil to cause silicone oil emulsification. Invest Ophthalmol Vis Sci 2014; 55(11): 7451-7456.

31 Sharma A, Grigoropoulos V, Williamson TH. Management of primary rhegmatogenous retinal detachment with inferior breaks. Br J Ophthalmol 2004; 88(11): 1372-1375.

32 Wickham L, Connor M, Aylward GW. Vitrectomy and gas for inferior break retinal detachments: are the results comparable to vitrectomy, gas, and scleral buckle? $\mathrm{Br} J$ Ophthalmol 2004; 88(11): 1376-1379.

33 Martínez-Castillo V, Zapata MA, Boixadera A, Fonollosa A, García-Arumí J. Pars plana vitrectomy, laser retinopexy, and aqueous tamponade for pseudophakic rhegmatogenous retinal detachment. Ophthalmology 2007; 114(2): 297-302.e291.

34 Alexander P, Ang A, Poulson A, Snead MP. Scleral buckling combined with vitrectomy for the management of rhegmatogenous retinal detachment associated with inferior retinal breaks. Eye (Lond) 2008; 22(2): 200-203.

35 Rush R, Simunovic MP, Sheth S, Chang A, Hunyor AP. 23Gauge pars plana vitrectomy versus scleral buckling versus combined pars plana vitrectomy-scleral buckling for medium-complexity retinal detachment repair. Asia Pacific J Ophthalmol 2014; 3(4): 215-219.

36 Totsuka K, Inui H, Roggia MF, Hirasawa K, Noda Y, Ueta T. Supplemental scleral buckle in vitrectomy for the repair of rhegmatogenous retinal detachment: a systematic review of literature and meta-analysis. Retina 2015; 35(11): 2423-2431.

37 Lincoff H, Kreissig I, Coleman DJ, Chang S. Use of an intraocular gas tamponade to find retinal breaks. Am J Ophthalmol 1983; 96(4): 510-516. 